drug can bind differently to these receptors and still have identical affinities for them. Additional structures (such as D3R or D4R in complex with risperidone) will probably be needed to answer this. Nonetheless, we expect that Wang and colleagues' D2R-risperidone structure, along with the previous D3R and D4R structures, will accelerate the design and discovery of D2R ligands that have higher selectivity than current antipsychotics, and potentially greater therapeutic impact.

David R. Sibley is in the Molecular Neuropharmacology Section, National Institute of Neurological Disorders \& Stroke,
National Institutes of Health, Bethesda, Maryland 20892-3723, USA. Lei Shi is in the Computational Chemistry and Molecular Biophysics Unit, National Institute on Drug Abuse, National Institutes of Health, Baltimore, Maryland 21224, USA. e-mails: sibleyd@ninds.nih.gov; lei.shi2@nih.gov

1. Perala, J. et al. Arch. Gen. Psychiatry 64, 19-28 (2007).

2. Wang, S. et al. Nature 555, 269-273 (2018).

3. Kebabian, J. W. \& Calne, D. B. Nature 277, 93-96 (1979).

4. Creese, I., Burt, D. R. \& Snyder, S. H. Science 192 , 481-483 (1976)

5. Seeman, P., Lee, T., Chau-Wong, M. \& Wong, K. Nature 262, 717-719 (1976)

6. Sibley, D. R. \& Monsma, F. J. Jr Trends Pharmacol. Sci. 13, 61-69 (1992)
7. Kapur, S. \& Remington, G. Biol. Psychiatry 50, 873-883 (2001).

8. Keck, T. M., Burzynski, C., Shi, L. \& Newman, A. H. Adv. Pharmacol. 69, 267-300 (2014).

9. Wang, S. et al. Science 358, 381-386 (2017).

10.Moritz, A. E., Free, R. B. \& Sibley, D. R. Cell. Signal. 41, 75-81 (2018)

11.Chien, E. Y. et al. Science 330, 1091-1095 (2010).

12.Newman, A. H. et al. J. Med. Chem. 55, 6689-6699 (2012).

13.Löber, S., Hübner, H., Tschammer, N. \& Gmeiner, P. Trends Pharmacol. Sci. 32, 148-157 (2011)

14.Carlsson, J. et al. Nature Chem. Biol. 7, 769-778 (2011).

15.Kapur, S. \& Seeman, P. Am. J. Psychiatry 158, 360-369 (2001).

16.Sykes, D. A. et al. Nature Commun. 8, 763 (2017).

17.Seeman, P. Clin. Neurosci. Res. 1, 53-60 (2001).

This article was published online on 26 February 2018.

\title{
Transistors driven by superconductors
}

\section{A hybrid transistor device has been made in which a superconductor forms a seamless interface with a semiconductor. The study of such interfaces could open the way to innovative applications in electronics. SEE ARTICLE P.183}

\section{YOSHIHARU KROCKENBERGER \& YOSHITAKA TANIYASU}

I ntegrating superconductors with semiconductors has long been thought to be essential to overcome the current limitations of electronic devices, but has been challenging to achieve. On page 183, Yan et al. ${ }^{1}$ report their use of a technique known as molecular beam epitaxy to grow layers of semiconductors on top of a superconductor. The resulting device has potentially useful electronic properties that hint at future applications for semiconductor-superconductor interfaces.

The development of increasingly sophisticated electronic devices is aided by efforts to make new combinations of materials - or, more specifically, new interfaces between materials, at which potentially useful electronic effects can occur. The credo underlying this concept is that "the interface is the

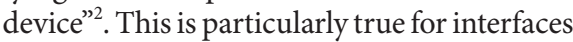
involving superconductors.

For example, Josephson junctions consist of two superconductors separated by a thin barrier, such as an insulator or a nonsuperconducting metal. Cooper pairs of electrons - the bound electron pairs that are responsible for superconductivity - can tunnel across the barrier in a fascinating physical process that has led to the development of devices such as those that mix or emit light at terahertz frequencies ${ }^{3}$. Interfacing superconductors with semiconductors ${ }^{4}$ such as indium arsenide (an arsenic-based material) can trigger Andreev reflection processes in which a normal electric current becomes a superconducting current. And if a ferromagnetic material (a material that exhibits the type of magnetism associated with iron) is used as the barrier in a Josephson junction, even more opportunities emerge for the manipulation of controllable electronic states $^{5}$.

Yan and colleagues now report the synthesis of interfaces formed between two a

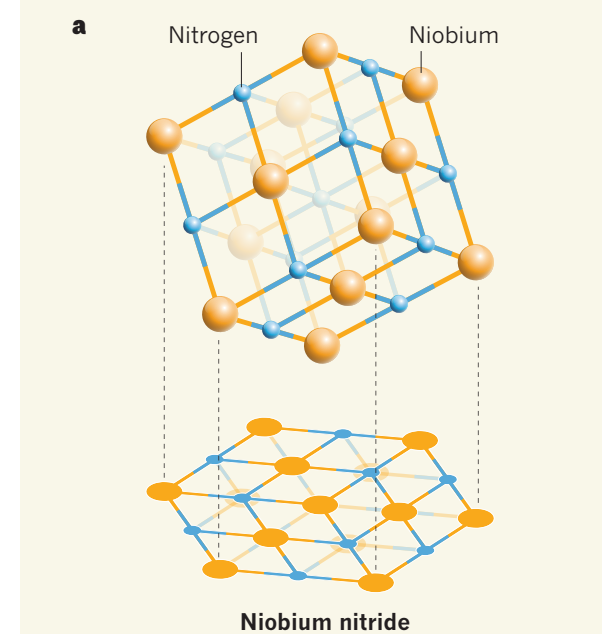

nitrides (nitrogen-containing materials), one a superconductor and the other a semiconductor. Nitride semiconductors ${ }^{6}$ are non-toxic, which makes them much more desirable for most applications than toxic arsenic-containing semiconductors. They can be synthesized in well-established procedures using molecular beam epitaxy - a technique in which atomized elements are deposited on a substrate in a vacuum to form thin films of single crystals. Nitride superconductors are also non-toxic, and, more importantly, are highly stable, particularly in ambient conditions (unlike many superconductors). The authors demonstrate that they can fabricate interfaces between a nitride superconductor and devices known as high-electron-mobility transistors ${ }^{7}$ (HEMTs) made from nitride semiconductors. HEMTs are widely used in communications infrastructures.

One problem that Yan and colleagues had to contend with is the fact that their nitride semiconductors have hexagonal crystal lattices, whereas the superconductor

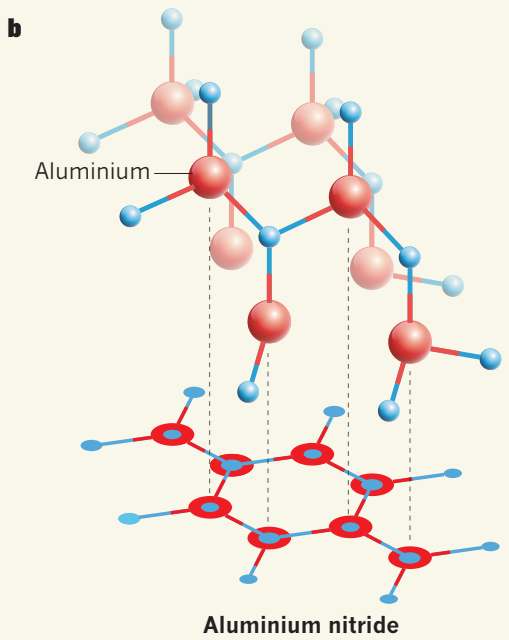

Figure 1 | Aligned views of materials that have different crystal lattices. a, The crystal lattice of the superconductor niobium nitride is cubic, but looks hexagonal when viewed from a particular orientation. b. The crystal lattice of the semiconductor aluminium nitride is hexagonal, and can therefore be aligned with the hexagonal arrangement shown in a. This allowed Yan et al. ${ }^{1}$ to prepare electronic devices in which a thin film of aluminium nitride is grown on top of niobium nitride, and the atoms of the two materials are aligned at the interface. 
(niobium nitride) is cubic (Fig. 1). This means that the crystallographic symmetry of their devices is broken at the interface between the cubic superconductor and the hexagonal semiconductor. Such broken symmetries can cause unwanted effects at interfaces, and therefore in devices.

This is where the orientation of the superconductor comes into play. Yan et al. grew a layer of the cubic superconductor on a substrate so that its lattice was oriented in a way that makes it look hexagonal. To picture this, imagine looking at a dice at an angle in which the diagonally opposite corners are aligned. What you see is a hexagon, even though the dice is cubic.

The same is true of the cubic superconductor on the substrate: a hexagonal arrangement of atoms is exposed on the surface, and the hexagonal semiconductor (aluminium nitride) aligns with this when it forms on top of the superconductor. As a result, the aluminium nitride is not perturbed by broken crystallographic symmetry at the interface, and forms an undistorted layer, as needed for the growth of an HEMT structure. Indeed, the authors observed the formation of certain quantum oscillations in their device; the presence of these oscillations is considered a benchmark of high crystal quality.

Yan et al. went on to measure the currentvoltage profile of their superconductor-HEMT structure. They observed that this profile of the HEMT is modified by a superconductor-tometal transition in niobium nitride, and generates a negative differential resistance (NDR) - a property that can be used to increase the power of electrical signals. NDR devices have been known since the end of the nineteenth century $^{8}$ and include the Gunn diode ${ }^{9}$, which is widely used to generate microwaves in sensors and measuring instruments. Such devices are of great value for electronic systems that use high-frequency, high-power signals - exactly what is needed in telecommunications networks. In Yan and colleagues' device, NDR can be switched on or off simply by making the temperature lower or higher than the critical temperature for superconductivity (the temperature below which superconductivity occurs).

Combining materials that have different electronic properties without breaking the crystallographic symmetry at the interface is a remarkable feat. However, the mobility of electrons in the device is currently rather low; much higher mobilities can be achieved in devices that use indium arsenide. Achieving mobilities comparable to those of indium arsenide will be extremely challenging. Moreover, the separation between the superconductor and the $2 \mathrm{D}$ electron gas - free electrons that are confined to move in only two dimensions generated in the device will need to be reduced to enable promising quantum effects.

A future goal could be to use the authors' system to generate and observe Majorana fermions ${ }^{10}$ - a type of quasiparticle that would be useful for quantum computing - at the superconductor-semiconductor interface ${ }^{11}$. Charge carriers in electronic devices can be scattered (for example, by crystal defects), and the average time between scattering events needs to be long to stabilize these quasiparticles. Yan et al. calculate that the charge-carrier scattering time in their devices is impressively long (66 femtoseconds; $1 \mathrm{fs}$ is $10^{-15} \mathrm{~s}$ ), but the scattering times will need to be at least 100 times longer, similar to the scattering time in indium arsenide ${ }^{12}$, to stabilize Majorana fermions. It remains to be seen whether this can be achieved in the authors' devices.

Ultimately, Yan and colleagues' work will inspire and accelerate efforts to grow nitride superconductors and nitride semiconductors that enable the ultra-high operating efficiency, structural perfection and opportunities for manipulating electronic properties that have already been achieved in interfaces involving indium arsenide. Because, at the end of the day, the interface is the device.
Yoshiharu Krockenberger and Yoshitaka Taniyasu are in the Materials Science Laboratory, NTT Basic Research Laboratories, Atsugi, Kanagawa 243-0198, Japan. e-mails:yoshiharu.k@lab.ntt.co.jp; taniyasu.yoshitaka@lab.ntt.co.jp

1. Yan, R. et al. Nature 555, 183-189 (2018).

2. Kroemer, H. Quasi-Electric Fields and Band Offsets: Teaching Electrons New Tricks (Nobel Lecture, 8 Dec 2000).

3. Tsujimoto, M. et al. Phys. Rev. Lett. 108, 107006 (2012).

4. Kjaergaard, M. et al. Phys. Rev. Appl. 7, 034029 (2017).

5. Senapati, K., Blamire, M. G. \& Barber, Z. H. Nature Mater. 10, 849-852 (2011).

6. Akasaki, I. Fascinated Journeys into Blue Light (Nobel Lecture, 8 Dec 2014).

7. Mimura, T. IEEE Trans. Microw. Theory Technique 50, 780-782 (2002).

8. Frith, J. \& Rodgers, C. Lond. Edinb. Dubl. Phil. Mag J. Sci. 42, 407-423 (1896)

9. Gunn, J. B. Solid State Commun. 1, 88-91 (1963)

10. Nichele, F. et al. Phys. Rev. Lett. 119, 136803 (2017).

11. Krogstrup, P. et al. Nature Mater. 14, 400-406 (2015).

12.Shojaei, B. et al. Phys. Rev. B 94, 245306 (2016).

\title{
EVOLUTION
}

\section{Mountains of diversity}

\begin{abstract}
A large-scale analysis of bird diversity and evolution on mountains around the globe explores the relationships between elevation, species richness and the rate of formation of new species. SEE LETTER P. 246
\end{abstract}

\section{ALEXANDER ZIZKA \& ALEXANDRE ANTONELLI}

$\mathrm{M}$ ountain chains are global centres of biological diversity - they harbour one-third of all terrestrial species ${ }^{1}$. These places have long fascinated biologists ${ }^{2}$, but are notoriously difficult to explore and study. Our knowledge of the distribution of species diversity on mountains is incomplete, as is our understanding of how species richness (the total number of species) and the rates of formation of new species (speciation) vary in single mountain ranges. On page 246, Quintero and Jetz ${ }^{3}$ tackle these issues by studying the diversity and evolution of birds on the 46 major mountain ranges of the world.

Mountains can differ substantially in the environment they provide, depending on factors such as bedrock, ruggedness, climatic conditions and the amount of energy available in the region. Moreover, mountains are often far apart, and organisms inhabiting such places can persist in genetically isolated populations owing to factors including terrain complexity and the high variation of habitat types along elevational gradients. Isolated populations often adapt to the local environmental and ecological conditions. When such populations are no longer capable of reproducing with one another, they form new species ${ }^{4}$. One example of this is the hummingbird Aglaiocercus kingii, which is found only in the Andes of South America (Fig. 1).

Quintero and Jetz used large-scale data sets of current distributions of bird species, mined from existing databases and publications, to characterize the relationship between elevation above sea level and species richness. The authors amalgamated data for 9,993 species, representing essentially all the birds that are currently known. Although the patterns observed in different regions vary, the overall trend for most regions is a hump-shaped curve in which species richness is highest at middle elevations, and decreases as elevation increases.

The result confirms findings from previous studies of plants and birds $s^{5,6}$. This type of pattern might be driven by the smaller area available for speciation at higher elevations and because the environmental conditions there are more extreme than those on lowlands. For example, large temperature fluctuations between day and night, and an increased exposure to radiation and wind at higher elevations could limit the number of species that can cope with such conditions.

The authors used some innovative approaches for their data analysis. They aimed 